\title{
Quantitative Electron Energy Loss Spectroscopy (EELS) Analysis of Flowable CVD Oxide for Shallow Trench Isolation of finFET Integration
}

\author{
J. Li ${ }^{1}$, J. Bruley ${ }^{2}$, R. Conti ${ }^{1}$, M. Belyansky ${ }^{1}$, S. Metha ${ }^{1}$, J. Strane ${ }^{1}$, L. Tai $^{3}$, L. Jiang ${ }^{1}$, J. Demarest $^{1}$, J. $^{2}$ \\ Gaudiello $^{1}, Y$. Zhu ${ }^{2}$, A. Grill ${ }^{2}$ \\ ${ }^{1}$ IBM Research, Albany, NY 12203; ${ }^{2}$ IBM Research, Yorktown Heights, NY 10598; \\ ${ }^{3}$ IBM Systems, Hopewell Junction, NY 12533
}

Email: juntaoli@us.ibm.com, Tel: (518) 292-7419

Non-planar semiconductor devices, such asvertical fin-based field-effect transistor (finFET) device, have become a viable technology for $22 \mathrm{~nm}$ node and beyond [1-4]. However, finFET device fabrication faces new challenges and process variation control becomes much more complicated. For instance, in the integration process of the finFETs, shallow trench isolation (STI) oxide recess is very critical to fin height control and has significant impact on the final electrical performance of device. This is because the channel height of a bulk finFET is defined by the junction isolation as well as the STI recess. The channel height uniformity is directly related to fin reveal or STI recess uniformity.

Due to the tight fin pitch, flowable CVD (FVCD) oxide has been introdued for void free oxide filling in between fins. Fin reveal by dry etch of oxide ususaly results in large across chip varibility due to dry etch loading effects. A wet etch (e.g., buffer HF) fin reveal process can also be used for STI oxide recess and has better variability control. However, recess using BHF etch is very sensitive to the incoming oxide material quality. In this paper, quantitative analysis of the elemental distribution within FCVD oxide with various post deposition treatments, such as ozone cure, Ultra-Violet cure, steam anneal, etc., and with varying aging time between cure and steam anneal has been investigated using Electron Energy Loss Spectroscopy (EELS) in a scanning transmission electron microscope with a dual EELS system. More specifically, the relative EELS quantification of major elements (silicon, nitrogen, oxygen) inside the FCVD oxide were performed. Fig. 1 shows the cross sectional TEM image of the FCVD oxide after ozone cure with no aging period before the TEM lamella was made. The as-deposited FCVD oxide quality is poor and contains "bubble" like low density regions in the gap between fins. This could indicate the FCVD oxide is not completely converted to pure oxide at this this point in the process. The EELS quantitative elemental maps from Fig. 2 confirm that the low density areas contain $15-20 \%$ N. The EELS map analyzed here covered $136 \times 148$ scanning pixels with a $1 \mathrm{~nm}$ pixel size a. Line profiles were extracted from the elemental maps along the yellow line from the same location in each map. Profiles integrated from 6 pixel ( $6 \mathrm{~nm}$ in width) are shown in Fig. 2. TEM and EELS characterization were also done on samples with ozone cure after extended self-aging time and a reduction in Nin the material was obsevered. This observation suggests that the FCVD oxide is unstable coming out of the ozone cure process. The film continues to age, and most probably reacts with oxygen/water from the ambient at room temperature prior to the final steam anneal process. To provide consistent density and composition, the film needs to age for about 10-15 hours in air in order to be completely converted by steam anneal. Another set of samples (with UV cured, 15 hours aging followed by steam anneal) show improved film quality. TEM evaluation does not reveal any nonuniform nanostrucutre (Fig. 3) in the oxide. Quantitative EELS analysis shows that, in this case, the filled oxide has converted into oxide with the $\mathrm{N}$ content being close to zero (Fig.4). In additon, EELS ionization cross sections will be calibrated using X-ray Photoelectron Spectroscopy (XPS) Argon ion depth profile analyiss of standard thermal oxide and silicon nitride blanket films 
In summary, quantitative EELS analysis of the FCVD STI oxide can be used to evaluate the oxide material quality within the STI trenches after various post deposition treatments.

\section{Reference}

[1] C. Auth, et al., Symp. VLSI Tech., p. 131, (2012).

[2] C.-H. Jan, et al., IEDM, p. 44, (2012).

[3] S.-Y. Wu, et al., IEDM, p. 224, (2013).

[4] T. Yamashita, et al., Symp. VLSI Tech., p. 14, (2011).

Acknowledgement: This work was performed by the Research Alliance teams at various IBM Research and Development Facilities.

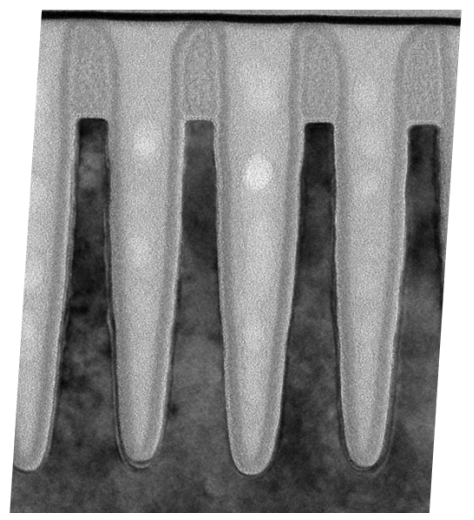

Fig.1 Cross-sectional TEM images of local STI filled with FCVD oxide after Ozone cure, zero aging time and steam anneal.
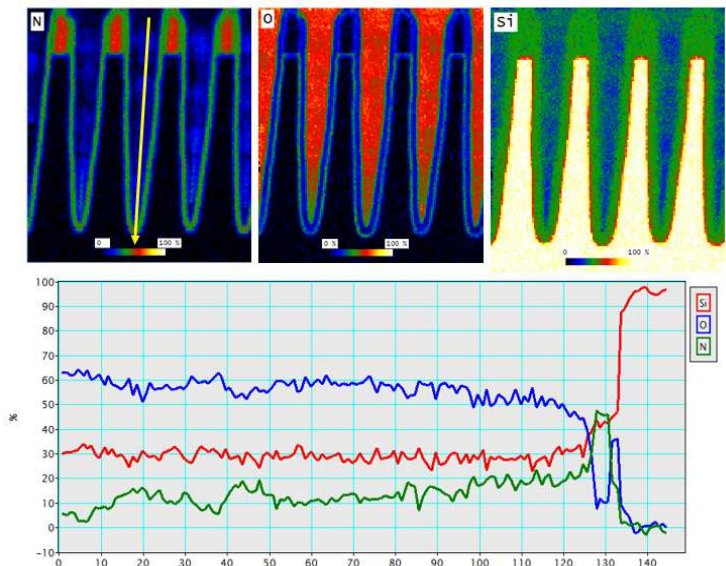

Fig.2 (top) Relative quantitative EELS Si, N and $\mathrm{O}$ maps; (bottom) The integrated line profile (6 pixels) extracted from the same location in each map data.

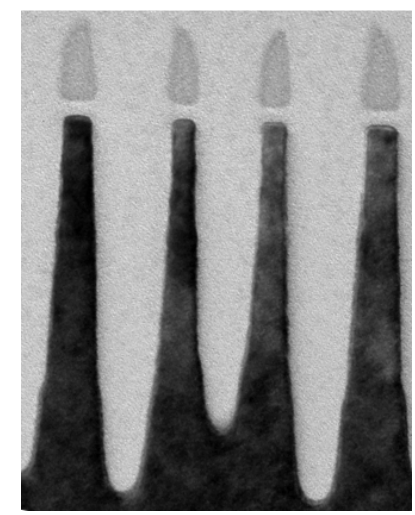

Fig.3 Cross-sectional TEM images of local STI filled with FCVD oxide after UV cure, 15 hour aging and steam anneal.
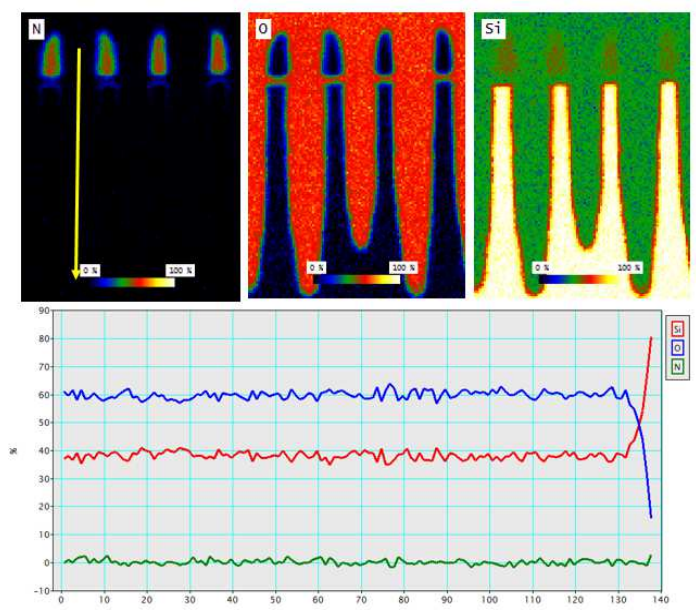

Fig.4 (top) Relative quantitative EELS Si, N and O maps; (bottom) The integrated line profile (6 pixels) extracted from the same location in each map data. 